\title{
Jarvik 2000 Implantation in Anatomical Right Ventricle on Patient with Corrected Transposition of Great Arteries
}

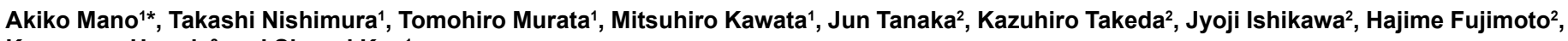
Kazumasa Harada² and Shunei Kyo ${ }^{1}$

${ }^{1}$ Department of Cardiothoracic Surgery, Tokyo Metropolitan Geriatric Hospital, Tokyo, Japan

${ }^{2}$ Department of Cardiology, Tokyo Metropolitan Geriatric Hospital, Tokyo, Japan

\begin{abstract}
The use of durable continuous-flow device for right ventricular (RV) remains limited and is evolving to date. We experienced a patient with corrected transposition of great arteries (CC-TGA) who needed ventricular assist device (VAD) for end-stage heart failure, was properly treated by Jarvik 2000 implantation in anatomical right ventricular.
\end{abstract}

Keywords: Durable ventricular assist device; Corrected transposition of great arteries; Anatomical right ventricle; Echo ramp study

\section{Introduction}

VAD has been recognized as an effective option for the patients with end-stage heart failure and it is usually implanted in left ventricle (LV). RV has much thinner wall, thus very high compliance, and much lower afterload compared to LV, so needs different management if VAD is applied. Congenital corrected transposition of great arteries (CC-TGA) is a rare anomaly which eventually progresses to systemic ventricular failure. Systemic ventricle in these patients is structurally right ventricle, however, has equivalent afterload to usual left ventricle. Thus, VAD implantation and management in these patients are challenging. We present a case of a 61-year-old gentleman, who was implanted Jarvik 2000 in anatomical RV, and was appropriately managed by careful echo ramp study and blood pressure control.

\section{Case Details}

A 61-year-old gentleman was transferred to our hospital due to severe heart failure. He was diagnosed with CC-TGA without concomitant cardiovascular anomalies, at age of 27 and underwent mechanical mitral valve replacement at age of 46 for severe mitral regurgitation. He also got permanent pacemaker for bradycardia after valve surgery. He had been doing well without any limitations of daily activities until age of 59, when he developed heart failure. $\mathrm{He}$ was started guideline directed medical therapy. However, his symptoms had been getting worse and became inotrope dependent at age of 61. He was referred to our hospital for advanced therapies. Despite maximal medical therapy and intraaortic balloon pumping, his condition continued to deteriorate, so we decided to implant VAD for bridge to transplantation. He got Jarvik $2000^{\circledR}$ (JARVIK HEART INC, NY) (Figure 1). An outflow cannula was connected to descending aorta by left-thoracotomy because of rotation of his heart and history of thoracotomy. The pump was placed into functional LV whose feature is consistent with RV (anatomical RV). Trabecular muscles were removed as much as possible to avoid dragging into inflow cannula and thrombus. Pump speed was set to dial $2(9,000 \mathrm{rpm})$ at the time of implantation. Post-operative course was uneventful. He was extubated on post-operative day (POD) 1 and was weaned-off inotropes on POD 10. Anticoagulation with intravenous heparin was started on POD 2 which was bridged to oral anticoagulation with coumadin on POD 3. The target international normalized ratio (INR) was set at 2 to 3 . An acetylsalicylic acid, at dose of $100 \mathrm{mg}$, was added for platelet inhibition. He started rehabilitation on POD 2. Initially, he tolerated exercise, however, complained dyspnea on effort as being increased exertion, so we evaluated echocardiogram (Figure 2). Functional LV end-diastolic dimension was $64 \mathrm{~mm}$ with fractional area change of $13 \%$. The ratio of end-diastolic dimension of functional RV and LV was appropriate from apical 4-chamber view, but estimated cardiac output measured at right ventricular outflow tract was insufficient $(2.5 \mathrm{~L} / \mathrm{min})$. Based on these findings, we changed pump speed from dial 2 to dial $3(10,000 \mathrm{rpm})$. At dial 3, the dimension of functional RV slightly increased, but geometry of both ventricles was maintained. Cardiac output increased to 3.5 $\mathrm{L} / \mathrm{min}$. Then, at dial $4(11,000 \mathrm{rpm})$, cardiac output was maintained. However, interventricular septum shifted to left side and functional
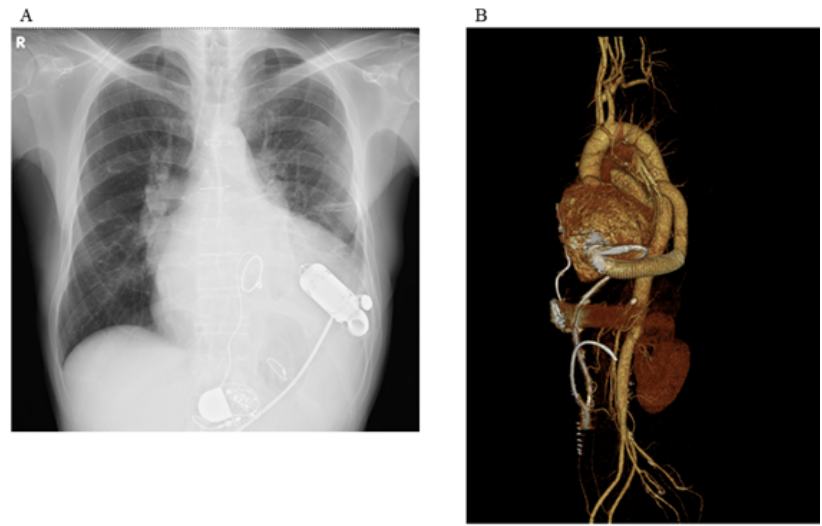

Figure 1: Chest radiograph (A) and 3-dimensional computed tomography (B) after Jarvik pump implantation.

*Corresponding author: Akiko Mano, MD, PhD, Department of Cardiothoracic Surgery, Tokyo Metropolitan Geriatric Hospital, 35-2 Sakae-Cho Itabashi-ku, Tokyo 173-0015, Japan, Tel: +81-3-3964-1141; Fax: +81-3-3964-4890; E-mail: akiko_mano@tmghig.jp

Received December 01, 2017; Accepted December 11, 2017; Published December 17, 2017

Citation: Mano A, Nishimura T, Murata T, Kawata M, Tanaka J, et al. (2017) Jarvik 2000 Implantation in Anatomical Right Ventricle on Patient with Corrected Transposition of Great Arteries. J Vasc Med Surg 5: 349. doi: 10.4172/23296925.1000349

Copyright: ( 2017 Mano A, et al. This is an open-access article distributed under the terms of the Creative Commons Attribution License, which permits unrestricted use, distribution, and reproduction in any medium, provided the original author and source are credited. 

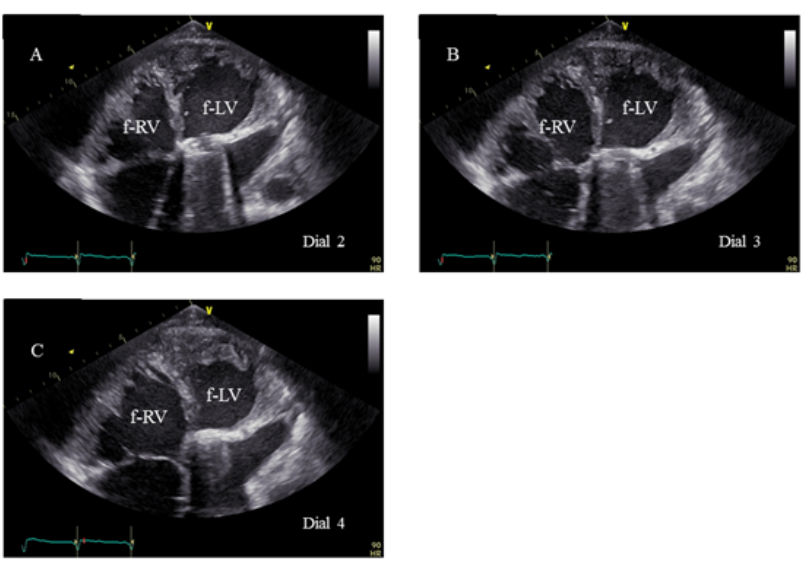

Figure 2: Echocardiograms after Jarvik pump implantation, at dial 2(A), 3 $(B)$, and $4(C)$. The ratios of functional right ventricular ( $f-R V)$ to functional left ventricular ( $f-L V)$ were acceptable range $(A$ and $B)$, but was disturbed $(C)$.

RV compressed functional LV, which suggested at risk of suction. At every pump speed, aortic valve showed almost full opening only during intermittent low speed (ILS). We concluded that the best pump speed was dial 3 for him on the basis of this ramp study. His symptoms on exercise resolved at this new speed. He continued to improve and tolerated further exertion. Repeated echocardiographic studies revealed good balance of biventricular end-diastolic dimensions and adequate cardiac output. For entire course, his mean blood pressure had been maintained under low 80'. We regularly checked free hemoglobin, bilirubin, haptoglobin, and lactate dehydrogenase blood levels, all of which had been within acceptable range. He had experienced neither thrombotic nor hemolytic events. Unfortunately, the patient developed massive intracranial hemorrhage on POD 109 and died on POD 110. We performed autopsy, which revealed no obvious thrombus inside heart as well as the pump.

\section{Discussion}

$\mathrm{VAD}$ is an established therapeutic option for the patients with endstage heart failure and the mainstream of VAD is currently durable continuous-flow devices. However, most VADs are applied to LV and durable VAD implantation in RV is not common [1,2]. RV has thinner wall, thus has much higher compliance and is very susceptible to pressure as well as to volume, compared to LV. The afterload against RV is also much lower because pulmonary vascular resistance is significantly lower than systemic vascular resistance. Therefore, different strategy should be considered when VAD is applied to RV. In our case, we implanted VAD in functional $\mathrm{LV}$, with anatomical structure of RV, but both afterload against ventricle and pressure gradient across VAD pump are almost same as normal LV. Thus, we focused on adequate systemic blood pressure control as regular LVAD, and did not need any modifications to increase afterload such as outflow graft banding, which is sometimes considered for right-sided VAD [3].
On the other hand, we carefully evaluated pump speed by repeated ramp studies. Functional LV was prone to be affected by speed change compared to normal LV due to higher wall compliance. Our concern was that usual pump speed might induce excessive functional LV unloading, which caused suction event. Furthermore, functional RV function was also reduced in our case. Generally, RV function should be closely monitored after LVAD implantation because excess LV unloading sometimes changes RV geometry, which leads to RV failure [4]. In this case, ramp study was very helpful to detect the best point to unload functional LV and keep functional RV geometry. We repeated ramp studies to confirm reasonable pump speed because we speculated that the patient might need further VAD support in accordance with physical recovery and more exertion. We were able to manage the patient on favorable pump speed and flow by ramp studies. There are several reports which showed successful VAD implantation in functional LV of patients with CC-TGA, however, most of them focused on operative procedures and few reports took note of the management based on echo study such as our report $[1,2,5]$.

In this case, it was possible to implant pump in functional LV apex with regular depth because functional LV had adequate size to be inserted it. Left-lateral approach was enough for good viewing. We removed trabeculae and tendon to minimize potential source of inflow cannula obstruction and thrombus, and actually didn't encounter any pump malfunction, thrombotic events, and hemolysis.

In conclusion, we implanted durable continuous-flow VAD in functional LV with anatomical feature of RV and systemic vascular resistance. Adequate blood pressure control and careful ramp study were useful to maintain appropriate pump flow without suction event and disturbance of biventricular geometry.

\section{Acknowledgement}

The authors thank Ms. Chieko Sakurayama and Mr. Teppei Maeda for their assistance with echocardiographic data collection and analysis.

\section{References}

1. Rajagopalan N, Booth DC, Diaz-Guzman E, Hoopes CW (2013) Successfu ventricular assist device placement in transposition of the great arteries with pulmonary hypertension. Ann Thorac Surg 95: e47.

2. Stewart AS, Gorman RC, Pocchetino A, Rosengard BR, Acker MA (2002) Left ventricular assist device for right side assistance in patients with transposition. Ann Thorac Surg 74: 912-914.

3. Timms D, Gude E, Gaddum N, Lim E, Greatrex H, et al. (2011) Assessment of right pump outflow banding and speed changes on pulmonary hemodynamics during biventricular support with two rotary left ventricular assist devices. Artif Organs 35: 807-813.

4. Kukucka M, Stepanenko A, Potapov E, Krabatsch T, Redlin M, et al. (2011) Right-to-left ventricular end-diastolic diameter ratio and prediction of right ventricular failure with continuous-flow left ventricular assist devices. J Heart Lung Transplant 30: 64-69

5. Huang J, Slaughter MS (2013) HeartWare ventricular assist device placement in a patient with congenitally corrected transposition of the great arteries. $J$ Thorac Cardiovasc Surg 145: 23-25. 